\title{
Agreement of bioelectrical impedance with dual-energy $X$-ray absorptiometry and MRI to estimate changes in body fat, skeletal muscle and visceral fat during a 12-month weight loss intervention
}

\author{
Kirsi H. Pietiläinen ${ }^{1,2 *}$, Sanna Kaye ${ }^{1}$, Anna Karmi ${ }^{3}$, Laura Suojanen ${ }^{1}$, Aila Rissanen ${ }^{4}$ \\ and Kirsi A. Virtanen ${ }^{3}$ \\ ${ }^{1}$ Obesity Research Unit, Department of Medicine, Division of Endocrinology, Helsinki University Central Hospital, \\ University of Helsinki, Biomedicum 1, Haartmaninkatu 8, PO Box 700, FIN-OOO29 HUCH, Finland \\ ${ }^{2}$ Institute for Molecular Medicine FIMM, University of Helsinki, Helsinki, Finland \\ ${ }^{3}$ Turku PET Center, Turku University Central Hospital, Turku, Finland \\ ${ }^{4}$ Obesity Research Unit, Department of Psychiatry, Helsinki University Central Hospital, Finland
}

(Submitted 16 January 2012 - Final revision received 8 June 2012 - Accepted 9 July 2012 - First published online 31 August 2012)

\section{Abstract}

The aim of the present study was to analyse the agreement of bioelectrical impedance analysis (BIA) compared with dual-energy X-ray absorptiometry (DXA) and MRI in estimating body fat, skeletal muscle and visceral fat during a 12-month weight loss intervention. A total of nineteen obese adults (twelve females, seven males) aged 20.2-48.6 years, mean BMI 34.6 (sE 0.6$) \mathrm{kg} / \mathrm{m}^{2}$, participated in the study. Body fat, skeletal muscle and visceral fat index were measured by BIA (Omron BF-500; Omron Medizintechnik) and compared with DXA (body fat and skeletal muscle) at baseline, 5 and 12 months, and with MRI (visceral fat) at baseline and 5 months. The subjects lost 8.9 ( $\mathrm{se} 1.8) \mathrm{kg}(9.0(\mathrm{se} 1.7) \%)$ of body weight during the 12-month intervention. BIA, as compared to DXA, accurately assessed loss of

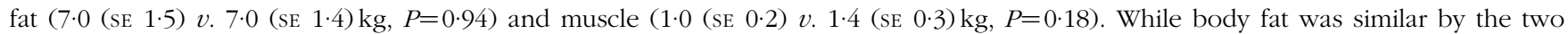
methods, skeletal muscle was underestimated by $1-2 \mathrm{~kg}$ using BIA at each time point. Compared to MRI, BIA overestimated visceral fat, especially in males. BIA and DXA showed high correlations for kg fat, both cross-sectionally and longitudinally ( $r$ 0.91-0.99). BIA, compared with DXA and MRI, detected kg muscle and visceral fat more accurately cross-sectionally $(r 0 \cdot 77-0 \cdot 87$ and $r 0 \cdot 40-0 \cdot 78$, respectively) than their changes longitudinally ( $r 0.24-0.61$ and $r 0.46$, respectively). BIA is at its best when assessing the amount or changes in fat mass. It is a useful method for measuring skeletal muscle, but limited in its ability to measure visceral fat.

Key words: Bioelectrical impedance: Body composition: Weight loss

Diagnosing obesity, the excess amount of fat that threatens health, is commonly performed by calculating BMI $\left(\mathrm{kg} / \mathrm{m}^{2}\right)$. However, the inability of this measure to distinguish between fat and lean mass and to determine fat distribution is well recognised. Assessment of body composition plays an important role, both in the clinical evaluation of obesity as well as in monitoring changes of fat and muscle mass during weight loss. It is of particular importance to avoid any loss of lean mass by negative energy balance, because this may suppress metabolic rate and facilitate post-dieting weight rebound $^{(1)}$.

Body composition measurements have only recently been used outside specialised obesity clinics and research units. The use of most advanced methods, such as MRI, computerised tomography or dual-energy X-ray absorptiometry (DXA) are mainly confined to research settings because of the high level of expertise required. The availability, cost (all of the aforementioned) and radiation exposure (computerised tomography and DXA) limit the use of these techniques at frequencies ideally needed for regular evaluation of the safety and efficiency of obesity treatment. Several simple, noninvasive and inexpensive methods, mainly based on bioelectrical impedance analysis (BIA), are now available also for the general public. It is therefore imperative to validate them in longitudinal body composition assessment.

In the present study, we examined the use of a tetrapolar BIA device in the assessment of body fat, muscle and visceral

Abbreviations: BIA, bioelectrical impedance analysis; DXA, dual-energy X-ray absorptiometry; FFM, fat-free mass; LTL, leg-to-leg; VLED, very-low-energy diet. 
fat against DXA and MRI during a 12-month weight loss intervention.

\section{Methods}

\section{Subjects}

A total of nineteen adults (twelve females and seven males) aged between 20.2 and 48.6 years (mean age: 35.8 (SD 7.7) years) participated in the study. The study group was recruited by newspaper advertisement and consisted of healthy obese weight-stable subjects. Exclusion criteria were smoking, weight change $>5 \mathrm{~kg}$ in the last 3 months, diabetes, endocrinological diseases or drug treatment affecting food intake or weight regulation. Of the subjects, eighteen were Caucasians and one CaucasianCaribbean, and all were resident in the Helsinki metropolitan area. The present study was conducted according to the guidelines laid down in the Declaration of Helsinki, and all procedures involving human subjects were approved by the local ethical committee of the Hospital District of Helsinki and Uusimaa. Written informed consent was obtained from all the subjects. The study was registered at clinicaltrials.gov NCT01312090.

\section{Protocol}

Subjects took part in a 12-month study, which consisted of individual and group-based diet and exercise counselling, starting with a 6-week modified very-low-energy diet (VLED) phase. All instructions were given and the measurements performed by the same nutritionist (L. S.). The VLED products were provided by Nutrilett and Allevo. In addition, the subjects were instructed to eat $0.5 \mathrm{~kg}$ vegetables for additional sources of fibre and vitamins, and protein-rich food snacks such as lean meat and milk products to yield a total of 70-90 $\mathrm{g}$ of protein daily during the VLED. After the VLED phase, a protein intake of $1.2-1.5 \mathrm{~g} / \mathrm{kg}$ was instructed for the patients. Multi-vitamins (Multi-Tabs, Orion Pharma) were provided for the subjects to ensure daily nutritional needs. Physical activity was recommended for the subjects according to the Finnish weight loss guidelines ${ }^{(2)}$. The subjects attended lifestyle counselling sessions bi-monthly until 5 months, whereafter the sessions continued once a month until the end of the study. To personalise the diet plans, individual sessions were given at months 0,2 and 5. Otherwise, the sessions were held in groups.

Measurements of body composition by BIA and a scale in a fasting state are reported in the present study at months 0, 2, 4,
5, 8, 10 and 12. DXA was measured at 0, 5 and 12 months and MRI at 0 and 5 months (Fig. 1).

\section{Anthropometry}

Fasting weight was measured barefoot and in light underwear to the nearest $0 \cdot 1 \mathrm{~kg}$ using a digital scale (Seca). Height was measured using a stadiometer to the nearest $0 \cdot 1 \mathrm{~cm}$ (Seca).

\section{Bioelectrical impedance analysis}

Body composition was assessed in a fasting state, after voiding, by a single-frequency BIA device, Omron BF-500 (Omron Medizintechnik), which uses eight electrodes in a tetrapolar arrangement that requires the subjects to stand on metal footpads in bare feet and grasp a pair of electrodes fixed on a handle with arms extended in front of the chest. The manufacturer's in-build pre-programmed equations were used to predict weight, body fat percentage, skeletal muscle percentage and visceral fat index ${ }^{(3)}$. Body fat $(\mathrm{kg})$ was calculated as 'body weight $(\mathrm{kg}) \times$ body fat percentage' and skeletal muscle $(\mathrm{kg})$ as 'body weight $(\mathrm{kg}) \times$ skeletal muscle percentage'.

\section{Dual-energy X-ray absorptiometry}

Assessments of fat mass and fat-free mass (FFM) were conducted using a whole-body DXA scan (Lunar Prodigy, software version 8.8) at standardised conditions, after voiding with no less than $4 \mathrm{~h}$ after a meal. For the calculation of muscle mass, an equation derived from Kim et al. ${ }^{(4)}$ was used. This equation uses appendicular lean soft tissue (lean mass from arms and legs) from DXA to derive skeletal muscle mass as follows:

$$
\begin{aligned}
& \text { total-body skeletal muscle } \\
& \qquad \begin{array}{l}
=(1.13 \times \text { lean mass from arms and legs })-(0.02 \times \text { age }) \\
+(0.61 \times \operatorname{sex}(0=\text { female }, 1=\text { male }))+0.97
\end{array}
\end{aligned}
$$

Percent age skeletal muscle was further calculated as follows: total-body skeletal muscle mass/body weight.

As a measure of central adiposity in DXA, $\mathrm{kg}$ fat in the android region was used according to the manufacturer's protocol. Android fat was estimated as the fat mass present in the area delineated by an upper horizontal border above the

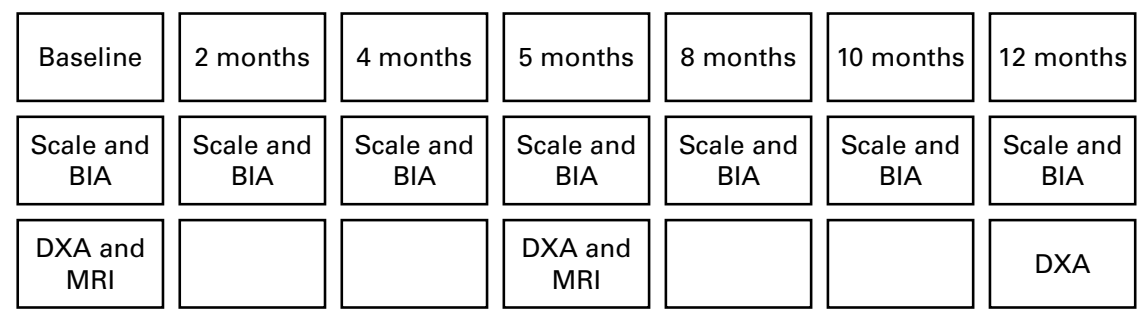

Fig. 1. Flow chart detailing the measurements performed at each time point. BIA, bioelectrical impedance analysis; DXA, dual-energy X-ray absorptiometry. 
pelvis line, at a position that was equivalent to $20 \%$ of the distance between the pelvis and the femoral neck, vertical borders lateral to the ribs and a lower border formed by the horizontal line passing through the top of the pelvis.

\section{$M R I$}

The abdominal region was imaged with $1.5 \mathrm{~T}$ Philips MRI scanner (Gyroscan Intera CV Nova Dual system, Philips Medical Systems) equipped with an internal body coil. Visceral fat volumes were assessed with SliceOmatic (version 4.3, TomoVision) and converted into fat weight using an adipose tissue density of $0.9196 \mathrm{mg} / \mathrm{ml}$.

\section{Statistical analysis}

Power calculations were based on measurement of body fat percentage and skeletal muscle percentage. For two methods with repeated measures (one baseline, six follow-up (2, 4, 5, 8, 10 and 12 months)), two-sided $\alpha 0 \cdot 05$, a power of $0 \cdot 90$, a correlation of 0.90 between baseline and follow-up measurements
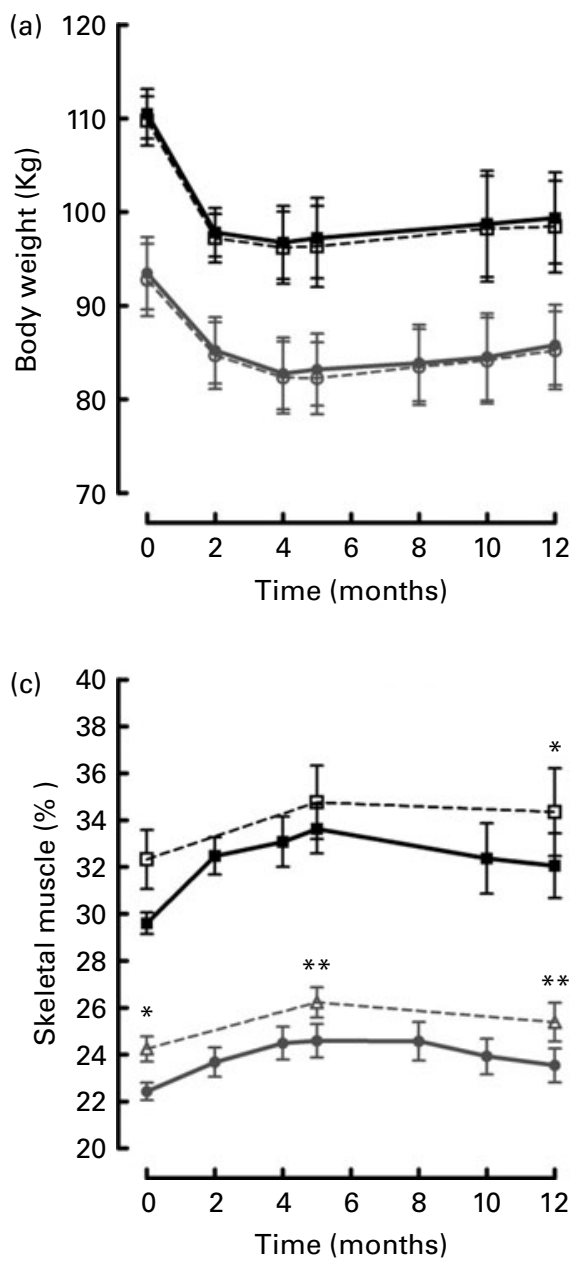

and correlation of 0.90 between follow-up measurements, the estimated sample sizes were: $n 14$ for estimation of body fat percentage and $n 18$ for estimation of skeletal muscle percentage. Males and females were matched for BMI. Wilcoxon's rank sum tests were applied to compare means of body composition measures by the methods and Mann-Whitney $U$ tests to compare males and females. Pearson's correlation coefficients adjusted for sex were used to test the relationship between results provided by BIA and the other methods. Agreement between the methods was further evaluated using Bland-Altman plots ${ }^{(5)}$. The Pitman's permutation test was used to compare the difference in variance for paired data ${ }^{(6)}$. All statistical tests were two-tailed and a $P$-value of $<0.05$ was considered statistically significant. Data were analysed using Stata software version 11.0 (Stata Corporation).

\section{Results}

At baseline, mean BMI was $34.6(\mathrm{SE} 0.6) \mathrm{kg} / \mathrm{m}^{2}$, not significantly different in males $(34.3$ (sE 0.9$\left.) \mathrm{kg} / \mathrm{m}^{2}\right)$ Compared
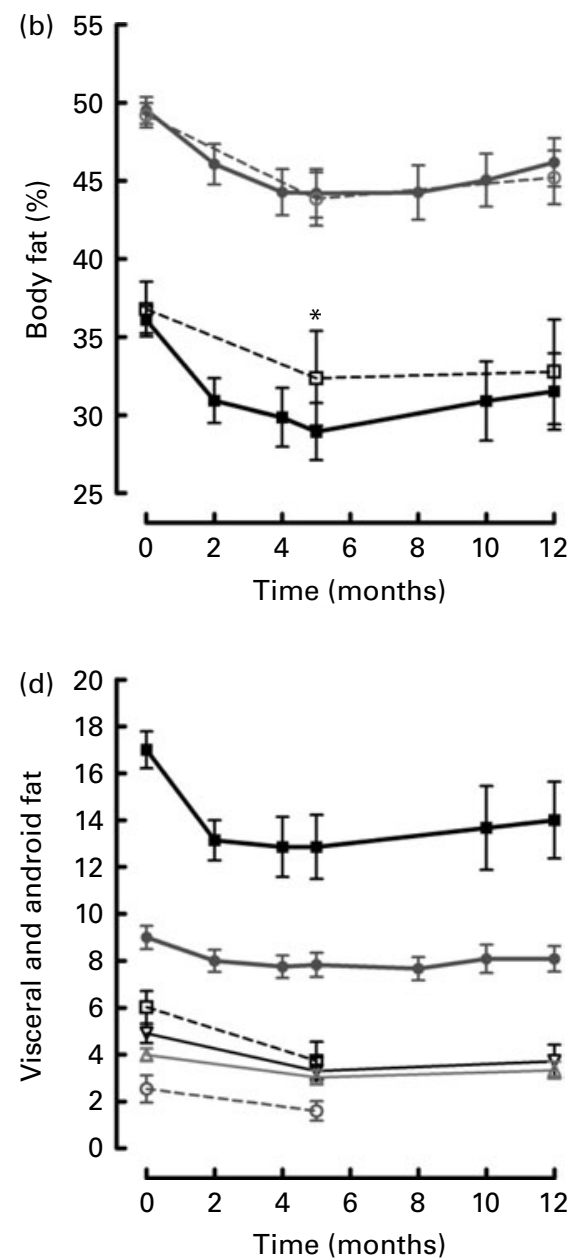

Fig. 2. Changes in (a) body weight, (b) body fat percentage, (c) skeletal muscle percentage and (d) visceral and android fat by each method during a 12-month weight loss intervention in nineteen subjects (seven males and twelve females). (a) _-, Males' bioelectrical impedance analysis (BIA); - $\boxminus-$, males' scale;

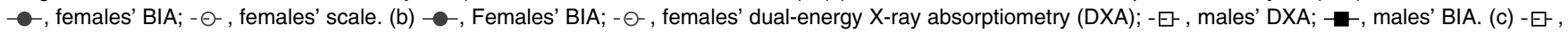
Males' DXA; - - males' BIA; - $\triangle$ - , females' DXA; - - , females' BIA. (d) - - , Males' BIA visceral index; - - , females' BIA visceral index; $\nabla-$, males' DXA android fat $(\mathrm{kg}) ; \triangle$, females' DXA android fat $(\mathrm{kg}) ;-\boxminus-$, males' MRI visceral fat $(\mathrm{kg}) ;-\Theta-$, females' MRI visceral fat $(\mathrm{kg})$. ${ }^{\star} P<0.05,{ }^{\star \star} P<0.01$. 
with females $\left(34 \cdot 8(\right.$ SE $\left.0 \cdot 8) \mathrm{kg} / \mathrm{m}^{2}\right)$. Measured by BIA and DXA, respectively, males $(40 \cdot 0$ (SE 1.8$)$ and $40 \cdot 7$ (SE 2.8) $\mathrm{kg}$ ) and females (46.5 (SE 2.5) and 45.3 (SE 2.3) kg) had comparable amounts of fat with no differences between the methods. Males had significantly larger skeletal muscle mass (32.7 (SE $0.5)$ and $35.3($ SE 1.1$) \mathrm{kg}$ ) than females (20.9 (SE 0.8) and $22.5(\mathrm{SE} 1.0) \mathrm{kg})(P<0.001$ between the sexes by both methods; BIA and DXA differed in females, $P=0 \cdot 0047)$.

Fig. 2 shows the patterns of change in weight and body composition measures by sex and by measurement method at each time point. During the first 5 months of the intervention, the mean weight loss was 11.6 (SE 1.3) kg, 11.8 (SE 1.2 ) $\%$ of body weight. BIA and DXA showed different losses of body fat $(10 \cdot 1(\operatorname{se~} 1.0) v .8 .9(\operatorname{se~} 1.0) \mathrm{kg}, P=0.027)$ and skeletal muscle $(0.5$ (SE 0.2) v. 1.5 (SE 0.4) kg, $P=0.010)$, respectively. Body fat percentage evaluated by BIA and DXA decreased by $6.0(\operatorname{se} 0.7)$ and 5.0 (SE 0.9$) \%(P=0.084)$ and skeletal muscle percentage increased by 2.9 (SE 0.4 ) and $2 \cdot 1$ (SE 0.4$) \%$, respectively $(P=0.053)$. Between 5 and 12 months, weight regained by 2.7 (SE 1.0$) \mathrm{kg}$, resulting in a net weight loss of 8.9 (SE 1.8 ) kg, 9.0 (SE 1.7 ) \% of body weight, at the end of the intervention. At 12 months, the subjects had lost comparable amounts of fat $(7 \cdot 0$ (SE 1.5) $v .7 \cdot 0$ (SE 1.4$) \mathrm{kg}, P=0.94)$ and muscle (1.0 (SE 0.2) $v$. 1.4 (SE $0 \cdot 3) \mathrm{kg}, P=0 \cdot 18)$ measured by BIA and DXA. Total losses of body fat percentage $(3.8(\operatorname{se} 0.9) v .4 .0(\operatorname{SE~} 1.0) \%, P=0.90)$ and increases in skeletal muscle percentage (1.6 (SE 0.5) v. $1.5(\mathrm{SE} 0.5) \%, P=0.55)$ were also comparable between the two methods. All of these changes were similar in males and in females.

With each method, males lost more visceral fat than females. Based on BIA, the visceral fat index decreased between baseline and 5 months by $4 \cdot 1$ (SE 1.0$)$ in males and 1.2 (SE 0.2$)$ in females $(P=0.0031)$, and between baseline and 12 months by 3.0 (SE 1.3) in males and $0.9(\operatorname{se~} 0.3)$ in females $(P=0.058)$. In MRI, visceral fat was presented in $\mathrm{kg}$, and decreased by $2 \cdot 4$ (SE 0.6) $\mathrm{kg}$ in males and $0.9(\operatorname{SE} 0.2)$ in females $(P=0.019)$ between baseline and 5 months (no measurement at 12 months). In DXA, the measure of central adiposity was $\mathrm{kg}$ fat in the android region. This measure decreased by 1.6 (SE 0.3$) \mathrm{kg}$ in males and by 1.0 (SE 0.1$) \mathrm{kg}$ in females by 5 months $(P=0.035)$, and by $1.2($ se 0.5$) \mathrm{kg}$ in males and by 0.7 (sE $0 \cdot 2) \mathrm{kg}$ in females by 12 months $(P=0 \cdot 18)$.

Comparability of BIA against the scale, DXA and MRI was assessed cross-sectionally and longitudinally by sex-adjusted correlations (Table 1) and Bland-Altman analyses (Table 2). Sex-adjusted correlation of weight measured by BIA and scale was close to 1.0 at all time points, both cross-sectionally and longitudinally (Table 1). Bland-Altman analysis showed that mean weights by BIA were $0.7-0.9 \mathrm{~kg}$ higher than by the scale cross-sectionally, but that both methods revealed similar mean weight changes with no significant differences in variance in the Pitman's tests (Table 2). For kg and body fat percentage, the correlations between BIA and DXA were significant at all time points. There was no significant bias of these measures cross-sectionally or longitudinally, and Pitman's tests showed good comparability of the methods with no differences in variances. BIA and DXA measures of 
skeletal muscle lacked correlation at baseline when expressed in percentages and when the change from baseline to 12 months was expressed in $\mathrm{kg}$. At all other time points, the correlations were high. Bland-Altman analyses showed that from baseline to 5 months, the decrease in skeletal muscle by BIA was on average $1 \mathrm{~kg}$ less than by DXA. At 12 months, the two methods no longer differed (Table 2).

Visceral fat index from BIA correlated with visceral fat from MRI at baseline only in females $(r 0.66, P=0.019)$, but not in males $(r-0.03, P=0.96)$. At 5 months, both sexes showed a high correlation ( $r 0.78$ in males and 0.81 in females). Significant correlations were found for the BIA visceral index and the android fat from DXA and waist circumference at all time points; however, they were lowest again at baseline (Table 1). Bland-Altman limits of agreement were not assessed, as each method had different units. Pitman's tests showed that at baseline $(r 0.63, P=0.004)$ and at 5 months $(r 0.80$, $P<0 \cdot 001$ ), BIA tended to overestimate the amount of visceral fat at high mean levels of visceral fat. On the other hand, with weight loss, BIA overestimated the loss of visceral fat when average levels of visceral fat loss in the two methods were large $(r$ 0.64, $P=0.004)$. This was more of a problem in males $(r 0.66, P=0.16)$ than in females $(r 0.161, P=0.62)$. The opposite was true, however, for BIA against DXA android fat (Table 2). Further, DXA android fat, as compared to MRI, overestimated visceral fat and loss of visceral fat more than BIA when the amount of visceral fat on average was large (data not shown). Of note, however, is that DXA android fat does not distinguish visceral from subcutaneous fat in this region.

In sum, BIA was at its best when estimating amounts and changes in weight, body fat percentage and skeletal muscle percentage. Visceral fat index was overestimated when the levels were high.

\section{Discussion}

There is a growing interest in the use of BIA to assess body composition during weight loss. As it is rapid, non-invasive and accessible, several recordings can be easily obtained as a function of time to evaluate the efficiency and safety of weight loss interventions. Wide availability of inexpensive BIA scales on the market makes its use increasingly common in self-monitoring body composition changes during weight loss in individual consumers. Therefore, the importance of the reliability and validity of the method cannot be overemphasised. In the present study, we examined the agreement of a single-frequency tetrapolar BIA with DXA and MRI during a 12-month weight loss intervention.

We have shown that BIA, as compared to DXA, accurately assessed body fat both cross-sectionally and longitudinally. In the present study, no differences were found for measurement of fat mass or body fat percentage by BIA and DXA at any time point, and correlations for the change in body fat during the 12-month intervention were up to 0.98. Jebb et $a l^{(7)}$ studied leg-to-leg (LTL) BIA, conventional tetrapolar BIA, DXA, air-displacement plethysmography and total body water in comparison to three- or four-compartment models 
in a 12-week weight loss intervention and subsequent follow-up at 1 year. The LTL-BIA served equally well as DXA, air-displacement plethysmography and total body water and better than tetrapolar BIA in estimating fat mass; comparisons with multi-compartment models showed correlations between 0.81 and 0.88 for LTL-BIA and between 0.36 and 0.47 for tetrapolar BIA during periods of weight loss and regain. Despite large standard deviations, mean differences in fat mass change in both BIA compared to multi-compartment models were at most $0.5 \mathrm{~kg}$. Utter et $a l{ }^{(8)}$ found that decreases in fat mass over a 12-week energy restriction were accurately detected by LTL-BIA, as compared to under-water weighing. Good agreement between fat mass from conventional tetrapolar BIA and DXA was also shown by Savastano et al. ${ }^{(9)}$ in patients before and at 12 months after laparoscopic adjustable gastric banding. Other studies have shown either under- ${ }^{(10,11)}$ or overestimation $^{(12)}$ of BIA fat mass, as compared to reference methods. In a 6-month study by Frisard et al. ${ }^{(10)}$, both LTL-BIA $(-2.6 \mathrm{~kg})$ and tetrapolar BIA $(-3.7 \mathrm{~kg})$ underestimated fat mass before weight loss and revealed correlations of 0.61 and 0.51 for detecting changes in fat mass by DXA and the two BIA, respectively. Thomson et al. ${ }^{(11)}$ found an underestimation of fat mass $(-3.8 \mathrm{~kg})$ by LTL-BIA, but not by multi-frequency BIA, before weight loss when compared with DXA. Reductions in fat mass over the 10-week weight loss intervention were tracked equally by both BIA, and regression coefficients with DXA were $0 \cdot 39-0 \cdot 40$. On the other hand, Linares et al. ${ }^{(12)}$ found that in a large population of 5740 subjects, the BIA significantly overestimated fat mass in comparison with DXA $(1 \cdot 1 \mathrm{~kg})$, and in seventy-two patients undergoing Roux-en-Y gastric bypass, BIA significantly overestimated changes in fat mass at 3 and 12 months. Estimates of changes in fat mass by each method were significantly correlated ( $\left.r^{2} 0 \cdot 4-0 \cdot 9\right)$.

In the present study, we calculated skeletal muscle from DXA by using previously established formulae ${ }^{(4)}$ in order to compare it to BIA, which in our case displayed skeletal muscle as the lean mass component. Using these DXA calculations as a reference, skeletal muscle mass was underestimated in BIA by $1-2 \mathrm{~kg}$ at each time point cross-sectionally and by $1 \mathrm{~kg}$ longitudinally between baseline and 5 months, but no longer at 12 months. Most other studies used FFM for the estimation of lean mass. These studies show large variations in the validity of FFM assessed by BIA systems during weight loss. In Utter et al.'s study ${ }^{(8)}$, the small changes in FFM over 12 weeks were equally effectively detected by LTL-BIA and under-water weighing. Savastano et al. ${ }^{(9)}$ reported small absolute differences and high $r^{2}(0 \cdot 82-0.99)$ during a 12-month study for tetrapolar BIA and DXA FFM, but validity of the change in FFM was not shown. In Powell et al.'s study of 32 weeks of weight loss ${ }^{(13)}$, LTL-BIA revealed similar mean values and changes in FFM to that of underwater weighing, but a systematic error of LTL-BIA underestimated FFM in subjects with higher FFM. Frisard et al. ${ }^{(10)}$ obtained higher estimates of FFM in LTL-BIA $(2 \cdot 7 \mathrm{~kg})$ and tetrapolar BIA $(3.0 \mathrm{~kg})$ than in DXA before weight loss. There was no correlation between DXA and LTL-BIA ( $\left.r^{2} 0 \cdot 09\right)$, but a larger correlation between DXA and tetrapolar BIA $\left(r^{2} 0.44\right)$ for the change in FFM. Thomson et $a l .{ }^{(11)}$ found an overestimation of FFM $(3.8 \mathrm{~kg})$ in LTL-BIA, but not by multi-frequency BIA before weight loss, when compared with DXA. After weight loss, LTL-BIA showed an increase in FFM by $2.1 \mathrm{~kg}$, while a decrease of $1.7 \mathrm{~kg}$ was observed for DXA and multi-frequency BIA.

Some of the above-mentioned studies assessed both fat mass and lean mass ${ }^{(8-11)}$, but none examined visceral fat. Many of the cited studies are old, and in the traditional tetrapolar BIA devices, this application was not available. The modern LTL-BIA measure electrical conductance from LTL, and therefore the bias towards the lower body may introduce imprecision to the measurement of visceral fat. Lack of wholebody analysis also applies to measurement of fat and FFM in LTL-BIA. We used a modern tetrapolar BIA device for the whole-body assessment. This device has been previously validated in a cross-sectional setting in weight-stable subjects ${ }^{(3)}$. In that study, the limits of agreement between visceral fat assessed by BIA and MRI were wide, and a systematic bias for underestimation of visceral fat at larger mean levels was observed for BIA. This is in contrast to the present study, which was limited to obese individuals, where BIA overestimated visceral fat when the average levels were high. Correlations for visceral fat by BIA and MRI were better $(r$ 0.92) in the previous ${ }^{(3)}$ than in the present study $(r 0.40-0 \cdot 78)$. Other cross-sectional studies have shown that visceral fat measured by BIA was lower than visceral fat by computerised tomography in patients with BMI $<35 \mathrm{~kg} / \mathrm{m}^{2}$, but higher in patients with $\mathrm{BMI} \geq 35 \mathrm{~kg} / \mathrm{m}^{2(14)}$. One possible explanation for the diverse results is the difficulty of any other method than MRI or computerised tomography to distinguish visceral from total abdominal fat. As men have more visceral fat relative to subcutaneous fat in the abdominal region than females, this may also introduce a sex-specific bias. These problems were evident in validity studies of new abdominal BIA devices ${ }^{(15-17)}$ While we did not find any longitudinal studies on visceral fat by BIA, our own study suggests that during weight loss, BIA overestimates the loss of visceral fat, especially in males, although due to small sample size, the sex-specific BlandAltman results were not significant in either sex.

The small sample size is an obvious limitation of the present study. With a larger sample size, it would have been possible to obtain more significant $P$ values of correlations (Tables 1 and 2) and narrow the width of the 95\% CI in the BlandAltman analysis (Table 2). In addition, males and females could have been analysed separately with greater possibilities to find statistical differences between the methods. Therefore, the results of the present study need to be interpreted carefully. However, the higher precision of longitudinal changes in body weight and fat percentage, the more modest agreement of skeletal muscle; and the low validity of visceral fat between the different methods is a result unlikely to change had we had a larger sample size. The present study represents a real-life situation of obesity clinics and introduces a possibility to use BIA on frequent terms in patient assessment. It does suggest that the clinical value of BIA lies in the measurement of body fat percentage, and any undue claims about its use in the measurement of visceral fat should be avoided. 
Reproducibility of BIA measures depends on several factors, including length of fasting, changes in hydration, previous food choices, drinking and exercise patterns. Therefore, the use of BIA in unsupervised settings needs careful attention. This may be the reason why BIA, as compared to the reference method DXA in the present study, revealed smaller correlations at baseline, when patients were less well aware of the strict requirements of the measurement, which they learned carefully during the course of the study. Based on wide variations in the previously published data, each BIA device would need its own validation study. Equations specific for sex, age and ethnicity are required, and it seems evident that obese subjects require additional consideration, especially when assessing visceral fat. More information is needed about the precision of visceral fat assessment by BIA during weight loss.

In conclusion, the results of the present study confirm the high level of preciseness of the Omron BF-500 (Omron Medizintechnik) BIA for predicting fat mass change during long-term weight loss. Skeletal muscle mass may be slightly underestimated by BIA, as compared with calculated estimations from DXA, but there was no systematic bias in the estimation in subjects with larger or smaller amounts of muscle tissue. Changes in skeletal muscle are better assessed in long- than in short-term periods. The visceral fat index captures the direction of change, but with systematic errors before and after weight loss, prediction of true visceral fat may be limited, especially in males.

\section{Acknowledgements}

We thank the participants for their invaluable contributions to the study. The study was supported by the Helsinki University Hospital Research Funds (K. H. P., S. K., A. R.), Turku University Hospital Research Fund (K. A. V.), a grant from Diabetes Research Foundation (K. H. P., K. A. V.) and grants from Novo Nordisk, Gyllenberg, Yrjö Jahnsson, Biomedicum Helsinki, Jalmari and Rauha Ahokas Foundation and the Finnish Foundation for Cardiovascular Research (K. H. P.). This research was designed by K. H. P. and K. A. V., and carried out by K. H. P., L. S., S. K., A. R. and K. A. V.; A. K. analysed the MRI data. K. H. P. drafted the manuscript and all authors accepted the final version. Conflict of interest: the BIA device and 3-month salary for L. S. and 2-month salary for A. K. were provided by Omron. The VLED products were provided by Nutrilett and Allevo, and multi-vitamins by the Orion Pharma.

\section{References}

1. Prentice AM, Goldberg GR, Jebb SA, et al. (1991) Physiological responses to slimming. Proc Nutr Soc 50, 441-458.

2. Working group appointed by the Finnish Medical Society Duodecim and the Finnish Association for the Study of
Obesity (2011) Current care guideline on obesity. http:// www.kaypahoito.fi/web/kh/suositukset/naytaartikkeli/ tunnus/hoi24010

3. Bosy-Westphal A, Later W, Hitze B, et al. (2008) Accuracy of bioelectrical impedance consumer devices for measurement of body composition in comparison to whole body magnetic resonance imaging and dual X-ray absorptiometry. Obes Facts 1, 319-324.

4. Kim J, Wang Z, Heymsfield SB, et al. (2002) Total-body skeletal muscle mass: estimation by a new dual-energy X-ray absorptiometry method. Am J Clin Nutr 76, 378-383.

5. Bland JM \& Altman DG (1986) Statistical methods for assessing agreement between two methods of clinical measurement. Lancet i, 307-310.

6. Neuhauser M \& Manly BF (2004) The Fisher-Pitman permutation test when testing for differences in mean and variance. Psychol Rep 94, 189-194.

7. Jebb SA, Siervo M, Murgatroyd PR, et al. (2007) Validity of the leg-to-leg bioimpedance to estimate changes in body fat during weight loss and regain in overweight women: a comparison with multi-compartment models. Int J Obes $\mathbf{3 1}$, 756-762.

8. Utter AC, Nieman DC, Ward AN, et al. (1999) Use of the legto-leg bioelectrical impedance method in assessing bodycomposition change in obese women. Am J Clin Nutr 69, 603-607.

9. Savastano S, Belfiore A, Di SC, et al. (2010) Validity of bioelectrical impedance analysis to estimate body composition changes after bariatric surgery in premenopausal morbidly women. Obes Surg 20, 332-339.

10. Frisard MI, Greenway FL \& DeLany JP (2005) Comparison of methods to assess body composition changes during a period of weight loss. Obes Res 13, 845-854.

11. Thomson R, Brinkworth GD, Buckley JD, et al. (2007) Good agreement between bioelectrical impedance and dualenergy X-ray absorptiometry for estimating changes in body composition during weight loss in overweight young women. Clin Nutr 26, 771-777.

12. Linares CL, Ciangura C, Bouillot JL, et al. (2011) Validity of leg-to-leg bioelectrical impedance analysis to estimate body fat in obesity. Obes Surg 21, 917-923.

13. Powell LA, Nieman DC, Melby C, et al. (2001) Assessment of body composition change in a community-based weight management program. J Am Coll Nutr 20, 26-31.

14. Berker D, Koparal S, Isik S, et al. (2010) Compatibility of different methods for the measurement of visceral fat in different body mass index strata. Diagn Interv Radiol 16, 99-105.

15. Thomas EL, Collins AL, McCarthy J, et al. (2010) Estimation of abdominal fat compartments by bioelectrical impedance: the validity of the ViScan measurement system in comparison with MRI. Eur J Clin Nutr 64, 525-533.

16. Browning LM, Mugridge O, Dixon AK, et al. (2011) Measuring abdominal adipose tissue: comparison of simpler methods with MRI. Obes Facts 4, 9-15.

17. Browning LM, Mugridge O, Chatfield MD, et al. (2010) Validity of a new abdominal bioelectrical impedance device to measure abdominal and visceral fat: comparison with MRI. Obesity (Silver Spring) 12, 2385-2391. 